\title{
Contribution to Real-Time Estimation of Crop Phenological States in a Dynamical Framework Based on NDVI Time Series: Data Fusion With SAR and Temperature
}

\author{
Caleb De Bernardis, Fernando Vicente-Guijalba, Tomas Martinez-Marin, \\ and Juan M. Lopez-Sanchez, Senior Member, IEEE
}

\begin{abstract}
In this study, a methodology based in a dynamical framework is proposed to incorporate additional sources of information to normalized difference vegetation index (NDVI) time series of agricultural observations for a phenological state estimation application. The proposed implementation is based on the particle filter (PF) scheme that is able to integrate multiple sources of data. Moreover, the dynamics-led design is able to conduct realtime (online) estimations, i.e., without requiring to wait until the end of the campaign. The evaluation of the algorithm is performed by estimating the phenological states over a set of rice fields in Seville (SW, Spain). A Landsat-5/7 NDVI series of images is complemented with two distinct sources of information: SAR images from the TerraSAR-X satellite and air temperature information from a ground-based station. An improvement in the overall estimation accuracy is obtained, especially when the time series of NDVI data is incomplete. Evaluations on the sensitivity to different development intervals and on the mitigation of discontinuities of the time series are also addressed in this work, demonstrating the benefits of this data fusion approach based on the dynamic systems.
\end{abstract}

Index Terms-Agriculture, data fusion, normalized difference vegetation index (NDVI), particle filter (PF), phenology, state space, synthetic aperture radar (SAR), temperature, time series.

\section{INTRODUCTION}

$\mathbf{R}$ EMOTE sensing data represent a profitable complement to agricultural management. During the last years, different technologies have provided alternative products related somehow to the biological status of crops. The knowledge of the current state of an agricultural land, updated with enough refresh rate and fine spatial resolution, is a powerful tool for practical management. This information can be exploited for a variety of products related to crops: yield prediction, hydrological requirements, optimum fertilisation time, etc. Estimation of specific dates, with special attention to the relevant events

\footnotetext{
Manuscript received November 06, 2015; revised February 06, 2016; accepted February 29, 2016. This work was supported in part by Spanish Ministry of Economy and Competitiveness (MINECO) and in part by EU FEDER under Project TEC2011-28201-C02-02 and TIN2014-55413-C2-2-P.

The authors are with the Institute for Computing Research, IUII, University of Alicante, E-03080 Alicante, Spain (e-mail: caleb@ua.es; fernando.vicente@ua.es; tomas@dfists.ua.es; juanma-lopez@ieee.org).

Color versions of one or more of the figures in this paper are available online at http://ieeexplore.iee.org.

Digital Object Identifier 10.1109/JSTARS.2016.2539498
}

from the agricultural point of view, has been proposed in [1][4]. In these studies, the normalized difference vegetation index (NDVI) is used to infer the phenological state of crops, and a measure of the crop productivity was proposed by Sellers et al. [5] using the same index. The NDVI represents then a suitable parameter in the estimation of the status of crops due to its relation with the biological process occurring in the plants. The index is defined as the ratio between the difference of the near-infrared (NIR) and red bands and the sum of these bands. The correlation between this variable and the biological process is due to both the chlorophyll contained in the leaves, which absorbs energy in the red spectrum, and their structure, which scatters the NIR components [6], [7].

Many satellite systems (e.g., LANDSAT, MODIS, or ASTER) provide regularly multispectral images of these bands with a large land coverage, thus making them suitable for agricultural purposes. The main difficulty of this observation scheme is the loss of acquisitions, due to internal problems, management considerations, maintenance, and other external causes. For instance, the presence of clouds might impede, depending on the specific area and period of cultivation, the development of a real-time operational product for phenological tracking. For this reason, relying on a single sensor technology does not guarantee the access to a regular time series of data. Accordingly, practical methodologies should be developed to be unaffected by temporal discontinuities, or at least to minimize their effect over the final product. Moreover, there are no technologies sensitive to every condition present in the crops along the whole phenological cycle. For example, multispectral optical sensors provide a good description on the radiation changes related to the plants' chemical variations, but they do not perceive structural or morphological variations. Considering other data from alternative technologies, as synthetic aperture radar (SAR) images, has a dual benefit: improving the sensitivity to the phenological development and increasing the temporal sampling for tracking the dynamics of the growth evolution.

In this context, data fusion is a very promising strategy for combining the information provided by different sources to improve the exploitation of the data. In remote sensing, data fusion has shown to be very effective to get better results 
in diverse applications [8], such as multitemporal image processing [9], change detection in urban areas [10], [11], land cover classification [12], estimation of elevation models [13], and vegetation monitoring [14]. Statistics-based methods are commonly used tools for implementing practical approaches for data fusion [15]-[17]. The particle filter (PF), within the dynamical approaches, is a suitable method to combine different information sources in statistical terms, with advantages over other techniques [18]. For instance, it does not make any assumption on the probability density function (pdf) of the sources. It also enables the integration of statistical information given by a physical or mathematical model that describes the behavior of the scene, independently of its nature.

In previous works, it has been shown that the use of the dynamical approach to estimate the phenology of rice crops presents high performance [19]-[21]. In the particular case of cereals, the growth cycle is usually defined in three main stages, namely vegetative, reproductive, and maturation. However, the temporal evolution along the different stages can be described identifying 10 main stages, which are subsequently divided into secondary stages. As a result, the scale derives in a continuous numerical scale of the plant development from 0 to 100 , using the extended $\mathrm{BBCH}$ scale [22]. The description in terms of the BBCH scale is based on the actual characteristics of an individual plant. When the scale is used for the definition of the development stage of a plant stand or a parcel or a field, the description should apply to at least $50 \%$ of the plants inside the parcel. Therefore, a specific field or parcel will be considered to reach a particular phenological stage when more than half of its plants reach that stage.

In the dynamical framework, the estimates of phenology are defined as state variables, evolving through a particular dynamical behavior characterized by a specific model called prediction model. The phenological states can be observed indirectly by different sources, e.g., remote sensing sensors. For a particular instant, the values predicted by the model and the values inferred from the different sensors can be combined, hence providing better results than if just one of them (model or observations) was considered. Moreover, this approach is carried out online or in real-time, i.e., an estimate is generated each time an observation is available. This is in contrast to other existing methodologies which provide estimates only after the end of the campaign.

In agricultural crops, air temperature is considered a dominant factor controlling the timing of different phenological states, and for that reason it has been widely used for predicting crop status [23], [24]. In particular, it has been pointed out as one of the main variables driving the phenological development in rice cultivations [25]-[27]. In this study, we have analyzed the incorporation of this information in the proposed dynamical methodology. The framework enables the integration of the temperature in a straightforward way, since it can be included in the prediction model as a control variable. In this manner, the particular conditions of the campaign under observation, represented by the accumulated temperature, are taken into account in the prediction model. In fact, this procedure represents an online improvement of the model as it is modified ad hoc for the specific conditions of each campaign.
In summary, the specific objective of this work is the development of a methodology to improve the estimation of the phenological state of rice crops by exploiting a fusion of data from time series of NDVI, SAR, and temperature values. For this purpose, a previously proposed dynamical approach is enhanced under a data fusion context. First, the observations given by NDVI are complemented by SAR acquisitions. Second, the prediction is also completed by a temperature driven model. The text is organized as follows. Section II describes the methodology, including the general theory and the particular aspects of the implementation used here. The available data set and the test site are introduced in Section III. Results are presented and discussed in Section IV. Finally, Section V summarizes the main points and the conclusion of this work.

\section{Methodology}

A dynamical system is a definition for any system that evolves over time. They are usually characterized by a set of first-order differential equations, which describes mathematically their physical behavior, as indicated in (1). The state vector $(\boldsymbol{x})$ contains all the information related to the status of the system at a particular instant. All the possible states define the state space that represents each and every condition the system can hold. Instead of regarding variables as functions of time, they can be thought as coordinates of points (states) in the $n$-dimensional domain the system is described in. One of the most relevant aspects of the dynamical methods relies on the fact that the future states are inherent to the model description

$$
\begin{aligned}
& x_{k+1}=f\left(x_{k}, u_{k}\right)+v_{k+1} \\
& y_{k+1}=h\left(x_{k+1}\right)+e_{k+1} .
\end{aligned}
$$

where a one-dimensional system will be considered hereafter. Equation (1a) describes the evolution of the state with time. In this notation, $x_{k}$ and $x_{k+1}$ represent the previous and the current (consecutive) states, respectively. Function $f()$ is known as the dynamic or prediction model, i.e., a representation of the expected behavior, relating $x_{k+1}$ with $x_{k}$ and the control variable $u_{k}$. The prediction error or uncertainty is characterized by the noise of the model $v_{k+1}$.

Usually, $x_{k+1}$ cannot be measured directly, but it can be inferred by external observations, e.g., using remote sensors. Equation (1b) relates the measured (noisy) observation $\left(y_{k+1}\right)$ to the current state $\left(x_{k+1}\right)$. Furthermore, $e_{k+1}$ represents the measurement error or uncertainty, which corresponds to stochastic noise process defined by its known pdf $p_{e_{k+1}}$. In this formulation, functions $f()$ and $h()$ do not depend explicitly on time $t$, hence resulting in a time-invariant system. Therefore, the estimation process is supported by the dynamics and not just by the experimental measurements (observations) themselves. In this context, all the noise sources are assumed to be nonstationary processes, since their parameters are extracted independently at each state during the modeling stage (see next sections for details).

\section{A. Data Fusion in a PF Context}

In this section, a brief summary of the PF is made with special attention to the data fusion methodology. A whole 
TABLE I

PF SEQUENCE
1) Initialization
2) Prediction
3) Update

4) Normalization

5) Resampling
Generate $N$ samples of $x_{0}$ from the initial pdf $p\left(x_{0}\right)$

Obtain the sample of $x_{k}^{i}$ from the transition pdf $p\left(x_{k+1} \mid x_{k}^{i}, u_{k}\right)$.

Evaluate the importance weights from the likelihood functions.

$\omega_{k+1}^{i} \propto \omega_{k}^{i} \prod_{j=1}^{M} p\left(y_{k+1}^{j} \mid x_{k+1}^{i}\right)$.

Normalise the weights $\omega_{k+1}^{i}=\frac{\omega_{k+1}^{i}}{\sum_{i=1}^{N} \omega_{k+1}^{i}}$.

The effective number of particles $\left(N_{\text {eff }}\right)$ provides a measure of

the number of particles with significant weight representing the

posterior pdf. If this number is lower than a provided threshold

$\left(N_{\text {thrd }}\right)$ the particles are redistributed with uniform weights to

the more likely values. description of the theory in particle filtering is presented in [28] and [29]. The use of alternative filters, such as the extended Kalman filter, has not been considered for this work. Although this filter is able to work under particular nonlinear models, the assumption of Gaussian noise process restricts its usage in our application, due to the multimodal nature of the observations. For this reason and the ability to work with any kind of nonlinear model, particle filtering provides a more appropriate framework [30].

$\mathrm{PF}$ is a sequential Monte Carlo method that approximates the posterior pdf with a set of particles, see (2). Particles represent tentative states in the state space following the evolution model (1a) and concentrate around the most probable state:

$$
p\left(x_{k+1} \mid y_{k+1}, u_{k}\right) \approx \sum_{i=1}^{N} \omega_{k+1}^{i} \delta\left(x_{k+1}-x_{k+1}^{i}\right)
$$

where $p\left(x_{k+1} \mid y_{k+1}, u_{k}\right)$ is the true posterior pdf, $x_{k+1}^{i}$ is the $i$ th simulated sample (particle), and $\omega_{k+1}^{i}$ is the weight of $i$ th simulated sample (particle). $N$ represents the number of particles employed in the simulation, and $\delta()$ is the Dirac delta function. If $N$ is sufficiently large, (2) approximates the true posterior pdf. The weights show the probability of each particle (state) at a particular instant $k$. The actual Monte Carlo simulation is applied over each one of the $N$ particles. The evolution model is assessed over each one of them in an iterative manner, i.e., the integration between $k$ and $k+1$ is divided in 1 -day steps. During the integration process, samples from the noise model distribution are included in the differential increments. These are updated recursively each time a new observation is acquired by the following equation:

$$
\omega_{k+1}^{i} \propto \omega_{k}^{i} p\left(y_{k+1} \mid x_{k+1}^{i}\right)
$$

where $p\left(y_{k+1} \mid x_{k+1}^{i}\right)$ expresses the likelihood function, and $y_{k+1}$ is the observation at instant $k+1$. Particles and observations are related by the observation equation (1b).
If more than one sensor is employed to provide measurements (observations) it is necessary to compute more than one likelihood function $p\left(y_{k+1} \mid x_{k+1}^{i}\right)$. Assuming that the observation sources are statistically independent, (3) can be rewritten to incorporate all likelihood functions as

$$
\omega_{k+1}^{i} \propto \omega_{k}^{i} \prod_{j=1}^{M} p\left(y_{k+1}^{j} \mid x_{k+1}^{i}\right)
$$

where $p\left(y_{k+1}^{j} \mid x_{k+1}^{i}\right)$ expresses the likelihood function for the observation given by the $j$ th sensor, and $M$ is the total number of sensors.

Finally, the current state $\left(\hat{x}_{k+1}\right)$ is obtained as a linear combination of all particles

$$
\hat{x}_{k+1}=\sum_{i=1}^{N} \omega_{k+1}^{i} x_{k+1}^{i} .
$$

A summary of each step involved in the algorithm is presented in Table I. The iterative process goes from Step 2) to Step 5), starting at Step 2) for each iteration. A complete description of the algorithm was presented in [20], so it is not repeated here to save space.

\section{B. Prediction Model for Crops}

In the case of rice crops, the model describing the evolution of the particles (prediction step, 2 in Table I) was presented in [21] and characterizes the phenological behavior of a particular set of rice fields. It is represented mathematically by a linear trend followed by a sigmoid function. The expression of the model using a discrete-time formulation is shown in the following equation:

$$
x_{k+1}=\left\{\begin{array}{cc}
x_{k}+m \Delta t, & x_{k}<x_{c} \\
x_{k}+r \Delta t\left(x_{k}-a\right)\left(b-x_{k}+a\right) / b, & x_{k} \geq x_{c}
\end{array}\right.
$$


where $\Delta t$ represents the integration time step, $t_{k}=k \Delta t$ in which $k$ takes integer values; $x_{k}$ is the phenological state at instant $k \Delta t$, and $x_{k+1}$ is the phenological state at the next instant; and $x_{c}$ is the phenological state at which the function is switched. Constants $m, r, a, b$, and $x_{c}$ are parameters of the function [21]. In this context, the current state can be calculated as a function of the previous state and the elapsed time.

Such a framework is open to add information in the prediction step to improve the value of the final outcome. For instance, data of the air temperature are well suited to fit into the model, representing a supplementary level of data fusion. The growing degree day $\left(\mathrm{GD}_{d}\right)$ is a heat index that is usually employed as an indicator of the phenological development of crops [25], [31]. The total or cumulated growing degree day $\left(\mathrm{CGD}_{d}\right)$ represents the integration over time of this variable during a growing season and can be computed using expression (7). Unless crops are extremely affected by droughts, pests or other unexpected events, the $\mathrm{CGD}_{d}$ can be used to predict when a crop will reach maturity

$$
\mathrm{CGD}_{d}=\mathrm{CGD}_{d-1}+\mathrm{GD}_{d}
$$

where

$$
\mathrm{GD}_{d}=T_{a}-T_{\text {base }}
$$

and

$$
T_{a}= \begin{cases}T_{\text {base }}, & \text { if } T_{a}<T_{\text {base }} \\ T_{\text {cutoff }}, & \text { if } T_{a}>T_{\text {cutoff }} \\ T_{\text {avg }}, & \text { otherwise. }\end{cases}
$$

In this model, $T_{a}\left({ }^{\circ} \mathrm{C}\right)$ represents the daily average growing temperature; and $T_{\text {base }}\left({ }^{\circ} \mathrm{C}\right)$ and $T_{\text {cutoff }}\left({ }^{\circ} \mathrm{C}\right)$ are the minimum and maximum temperatures for physiologic crop growth, respectively. $T_{\text {avg }}$ is the average of the daily temperature $\left(T_{\mathrm{avg}}=\left(T_{\max }+T_{\min }\right) / 2\right)$, in which $T_{\max }$ and $T_{\min }$ are the maximum and minimum daily temperatures.

With the objective of including the information provided by the temperature, the prediction model can be evaluated including a control variable $\left(u_{k}\right)$ as expressed in (1a). Therefore, the model provides the evolution defined by $f()$ but subject to the variable $u_{k}$, which represents the influence of $\mathrm{CGD}_{d}$ in the prediction. In particular, the daily average temperature $\left(T_{\text {avg }}\right)$ is considered as the control variable, i.e., the input for the prediction model. A continuous temperature time series is used for computing a prediction estimation in the same temporal sampling as the model.

The action of this variable governs the velocity of the phenological development in the prediction step. The phenological evolution model is determined by the combination of the average model and the effects of the accumulated temperature. In this context, local variations on the control variable would affect directly the phenological evolution rate. To derive the model driven by $\mathrm{CGD}_{d}$, a particular set of reference parcels can be employed to characterize the phenological behavior in terms of this variable. As illustrated in Fig. 1, the prediction model in this case can be obtained after fitting a polynomial function to the relation between the $\mathrm{CGD}_{d}$ and the phenological information over the reference set.

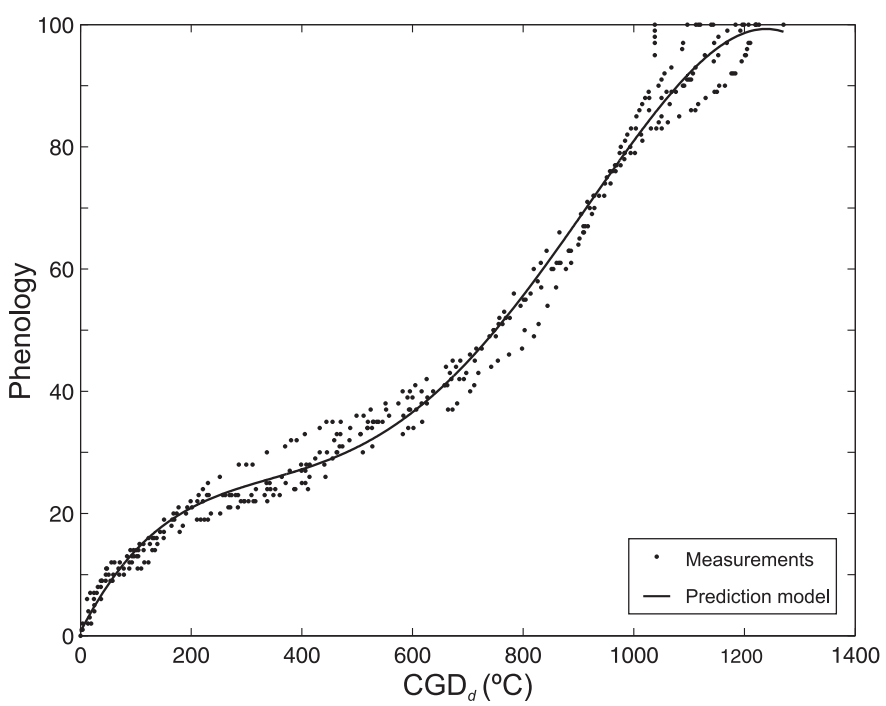

Fig. 1. Relation between the cumulative growing degree days $\left(\mathrm{CGD}_{d}\right)$ and the reference phenological values (dots) for 2008-2013 (except 2012 due to lack of ground data). The derived function is obtained from the fitting of a polynomial function (line) (degree $=4)$. The calibration parameters $\left(T_{\text {base }}, T_{\text {cutoff }}\right)$ for the $\mathrm{CGD}_{d}$ model are extracted from [26].

\section{Observation Model}

In this work, the observation of the scene is carried out by two different sensors $(M=2)$ at the same or different time: the first one provided by a multispectral image (NDVI product) and the second from a SAR acquisition (HH/VV ratio). Therefore, instead of only one equation, two models are necessary in this case to compute the different variables and to provide the likelihood functions appearing in (4) (update step, 3 in Table I).

1) NDVI Observation: The relation between the phenological state and the particular value of NDVI for each particle is given by (10), which corresponds to a double-logistic curve. It has been extracted from [21] and included here for completeness

$$
\begin{aligned}
y_{k+1}^{1}= & c+d\left[\frac{1}{1+\exp \left(-r_{1}\left(x_{k+1}-f_{1}\right)\right)}\right. \\
& \left.+\frac{1}{1+\exp \left(-r_{2}\left(x_{k+1}-f_{2}\right)\right)}-1\right]+e_{k+1}^{1}
\end{aligned}
$$

where $c$ is the minimum NDVI value and $c+d$ the maximum NDVI value. $r_{1}$ and $f_{1}$ are parameters of the first logistic curve, $r_{2}$ and $f_{2}$ correspond to the second logistic curve, and $e_{k+1}^{1}$ represents the observation noise. In Fig. 2, the NDVI measured in a set of parcels from 2008 to 2013 (excluding 2012 due to lack of ground data) and the fitting made by (10) are presented. More details about this model can be found in [21].

2) SAR Observation: A polarimetric synthetic aperture radar (PolSAR) image provides different products (power of the different polarimetric channels, correlations and phase differences between them, etc.) [32]. This is analogous to the multispectral case, in which different bands capture different ranges of the spectrum.

An analysis of a wide set of polarimetric variables as a function of the phenological evolution of rice fields can be consulted 


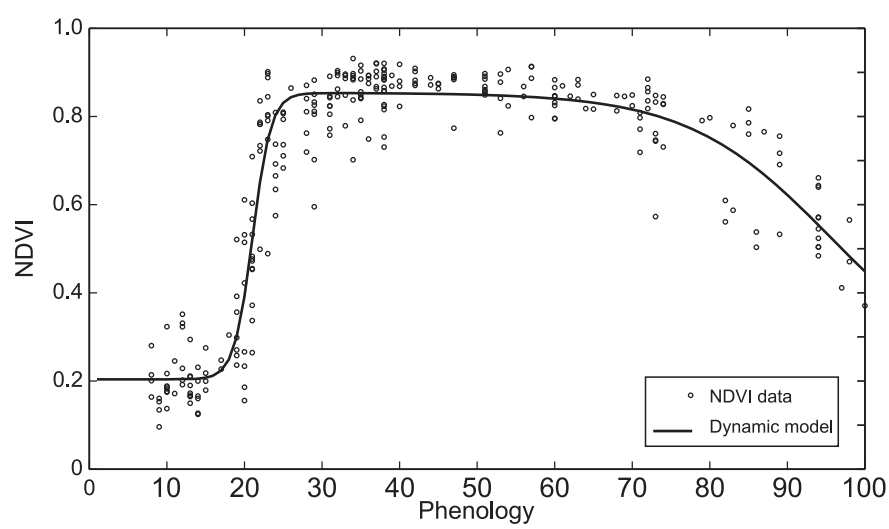

Fig. 2. Measurements of NDVI (observations) for different parcels from 2008 to 2013 (excluding 2012 due to lack of ground data) represented as a function of phenological state (black circles). The solid line corresponds to the observation equation for the optical system with $r_{1}=0.84, f_{1}=21.07, r_{2}=-0.10$, $f_{2}=95.40, c=0.21, d=0.65$. Extracted from [21].

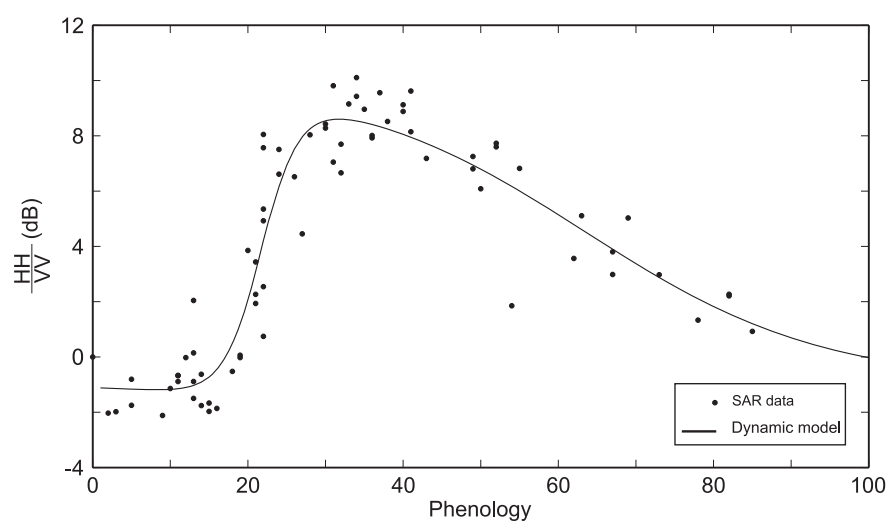

Fig. 3. Measurements of the ratio HH/VV (dB) for a set of parcels between 2008 and 2009 represented as a function of phenological state (black circles). The solid line corresponds to the observation equation, i.e., the HH/VV value for each phenological state given by (11) with $r_{1}=0.39, f_{1}=21.69, r_{2}=$ $-0.06, f_{2}=63.38, c=-1.01, d=11.12$.

in [33] and [34], for C- and X-band, respectively. From these studies, and from previous works which exploited SAR images for rice mapping [35], we have selected the ratio between the two backscattering coefficients of the copolar channels $(\mathrm{HH}$ and VV) as a measure sensitive to the crop development. As a key point for our purposes, this ratio exhibits a clear evolution in the phenological range from state 30 to 70 . Consequently, this observation increases the sensitivity provided by the NDVI data during the phenological range from 30 to 70 , i.e., where NDVI is almost constant (see Fig. 2). However, there is no constraint in the use of any other radar or polarimetric variable, as far as it increases the sensitivity to the phenological development. In our case, the HH/VV ratio is computed from a stack of TerraSAR-X images acquired with an incidence angle of $30^{\circ}$, which is described in [34].

In Fig. 3, the HH/VV ratio measured in a set of parcels in 2008 and 2009 is shown. With the same method followed to derive the equation relating phenological states with the NDVI, an equation can be derived for the relation between phenology and the HH/VV ratio. It is observed in Fig. 3 that the data can be fitted well also by a double-logistic curve

$$
\begin{aligned}
y_{k+1}^{2}= & c+d\left[\frac{1}{1+\exp \left(-r_{1}\left(x_{k+1}-f_{1}\right)\right)}\right. \\
& \left.+\frac{1}{1+\exp \left(-r_{2}\left(x_{k+1}-f_{2}\right)\right)}-1\right]+e_{k+1}^{2}
\end{aligned}
$$

where $c$ is the minimum $\mathrm{HH} / \mathrm{VV}$ value and $c+d$ the maximum, $r_{1}$ and $f_{1}$ are parameters of the first logistic curve, $r_{2}$ and $f_{2}$ correspond to the second logistic curve, and $e_{k+1}^{2}$ represents the observation noise.

\section{Noise Modeling Considerations}

To apply the proposed methodology, the noise distribution of each model in (1a) and (1b), i.e., $v_{k}$ and $e_{k}$, has to be fully characterized by its pdf. Providing an analytical expression for these pdf's is unfeasible. Consequently, they are computed from the fitted model and the individual observations of the modeling step. In order to accomplish that, it is assumed that every noise distribution behaves as a nonstationary Gaussian process which evolves along the model. The parameters of the distributions are computed for each state with significant number of samples in the model, and those with no samples are interpolated. The mean of the distribution is provided by the previously fitted model, and the variance is obtained by analysing the scattering of the samples in that state. Therefore, the variability of each of the measurements involved (phenological ground truth and observations) is immersed in the estimation scheme. Simply stated, it allows a representation of the confidence level on the model value. This procedure is applied over each model to derive its own particular evolutionary pdf, which is employed afterward in the exploitation of the algorithm for estimation purposes.

\section{E. Analysis Method}

Although there exist alternative methodologies for crop phenological estimation, they are based on the estimation of a very reduced set of stages (4-5) [33], [34], [36], [37] hence the comparison of the proposed technique with these previous ones is not necessary. The objective of the proposed analysis is to assess that the incorporation of additional sources of information is useful to reduce the error obtained in the case that only considers the temporal series of NDVI images.

The described methodology has different applications under the same context. For instance, the same approach can be employed to estimate the date of arrival of a particular state, i.e., prediction of dates. However, this evaluation is not included in this study and we have focused the analysis on the estimation of the phenological state for a given date. In order to validate the improvement given by the SAR observations and the incorporation of temperature on the prediction model, the results are divided in two groups. The first one corresponds to the effects of the data fusion with SAR acquisitions (Section IV-A). In this case, the prediction model is based just on time evolution. The phenology is inferred in three ways using: 1) exclusively 

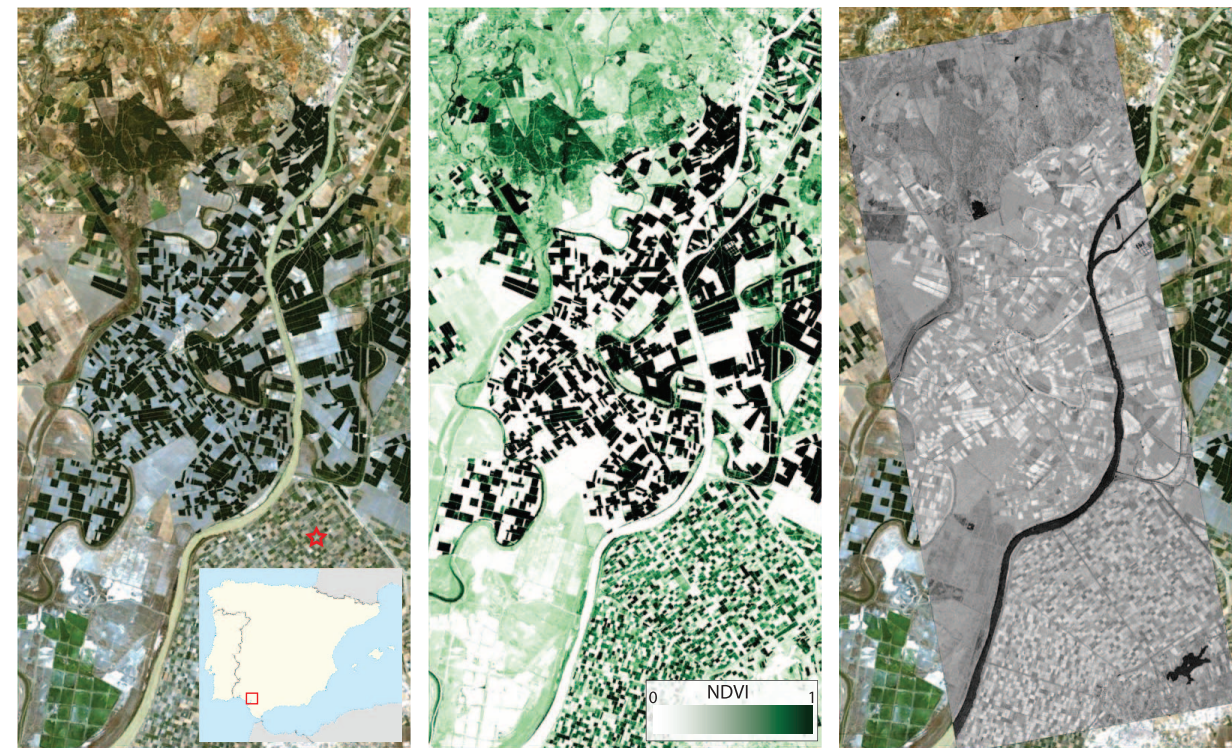

Fig. 4. Overview and location of the area under analysis. The scene is located in the Southwest of Spain, covering an approximate area of $30 \times 30 \mathrm{~km}$. The left image corresponds to the visible map from Landsat-7 and the precise location over the Iberian Peninsula. The middle image is the NDVI derived product from the same sensor. The right image represents an overlay of the corresponding TerraSAR-X image [HH (dB)] over the visible map. Images were acquired within consecutive days, August 3, 2008 and August 4, 2008 for Landsat and TerraSAR-X, respectively. The red star on the visible image indicates the temperature ground station location.

the NDVI time series for each parcel; 2) NDVI in combination with SAR data; and 3) based only on SAR data. The analysis can be divided in specific ranges to detect whether different sensitivities are detected or not. Moreover, dealing with remote sensor images is likely to have image drops or gaps between acquisitions which can derive in discontinuities on the temporal series. An evaluation is also performed in this sense, i.e., with removal of images, to explore the behavior of the approach under these circumstances. In the second part, the same three evaluations are carried out by employing the prediction model which incorporates the temperature information (Section IV-B).

Regarding the particular aspects of the estimation, the total number of PF employed for the test is $N=5000$ and the resampling threshold is fixed to $N_{\text {thrd }}=1000$ particles. In order to speed up the convergence of the estimation process, it is assumed that the first observation always is provided in the first part of the phenological range. Thus, $p\left(x_{0}\right)$ is described by a uniform distribution of particles between states 0 and 40. Alternatively, a uniform distribution along the whole range (0-100) could be used, at the cost of sacrificing convergence time.

To provide a final value, after each iteration in the estimation approach the average value of the posterior pdf is selected as estimation. Alternatively, the peak of the distribution could be also selected, but in our tests both options have yielded similar results. It is important to clarify that we may encounter multimodal distributions in this algorithm, which are related exclusively to the observation model since the early and late stages tend to behave in similar ways (i.e., similar NDVI values). Considering a single observation in one of these ranges derives in a multimodal distribution, almost impossible to relate to one or the other with certainty. Nevertheless, the support of a dynamical model, i.e., prediction, allows us to remove the multimodal effects on the estimated posterior pdf.

\section{Data Set and Test Site}

This methodology has been tested with data acquired over rice fields located in Seville, Southwest of Spain, during 2008 and 2009. An overview of the study area is shown in Fig. 4. In this area, rice is cultivated from May to October (during 135-150 days), with just one crop per year. The phenological information, acquired by ground campaign measurements, was provided by the local association of rice farmers (Federacion de Arroceros de Sevilla) on a weekly basis more than nine parcels in 2008 and 10 parcels in 2009. In addition, the sowing and harvest dates are known for each parcel. This information was used in the generation of the model and in the validation of the results. The employed parcels in the analysis range from 3 to 17 ha, with plant densities from 200 to 850 plants $/ \mathrm{m}^{2}$.

The number of NDVI images that has been used is 12 for 2008 and 8 for 2009. All the NDVI images employed in this work were downloaded from the Servidor de imágenes Landsat y productos derivados de Doñana (http://mercurio.ebd.csic.es/imgs/), a web server maintained and operated by the Laboratorio de Sistemas de Información Geográfica y Teledetección de la Estación Biológica de Doñana, LAST-EBD, CSIC, Spain. The images were acquired by Landsat 5 and Landsat 7 with a pixel size of $30 \times 30 \mathrm{~m}$. All the images were already geometrically and radiometrically corrected [38], [39]. The SAR images were acquired by the TerraSAR-X with a 11-day revisit period. The number of employed acquisitions is 11 for 2008 and 10 for 2009. This data set is described in depth in [34]. 
The temperature information is obtained from the meteorological station La Puebla del Río II of the Spanish Sistema de Información Agroclimática para el Regadío (SIAR) (see location at Fig. 4). The distance from the meteorological station to the farthest crop field in this scenario is less than $24 \mathrm{~km}$. According to the information provided by the local authorities, we can assume a very low spatial frequency behavior for temperature (differences between fields are always well below $1{ }^{\circ} \mathrm{C}$ ), which means that we consider the same temperature values for all the parcel set.

In order to define a common spatial resolution, the observations from each sensor are computed at parcel level, i.e., a single observation is generated for each parcel. Evidently, this approach degrades the optimal spatial resolution, but it enables to combine the finer remote sensing resolution with the ground truth relative to the phenological state, provided at parcel level. However, the methodology could be extended without any change to a finer spatial grid. On the other hand, if a SAR sensor with coarser resolution were used (e.g., Sentinel-1), the approach working at parcel level would be perfectly applicable since the parcel size is large enough to ensure a sufficient number of looks in the measurements.

\section{RESUlts AND Discussion}

\section{A. Results Considering Only Remote Sensing Data}

1) NDVI Time Series: For illustration purposes, the estimates obtained for one representative parcel are presented in Fig. 5 using in this case just the NDVI time series as observation data. Each time an image is available, prediction and observation are combined by the PF to provide the current state (solid circle). When the acquisition is not available, due to the presence of clouds or other system failures, the estimation is limited to the predicted value (empty circles). For validation purposes, the ground truth data for the parcel under study are shown by a dashed line. The estimation series for this parcel consists of a total of 17 estimations ( 8 based on NDVI and prediction and 9 based exclusively on prediction) with a temporal base of 8 days, producing a root-mean-square error (rmse) of 11.2 states.

It can be observed how after three acquisitions ( 24 days after the first one) a satisfactory convergence to the state of the parcel has been reached. The prediction model warrants to obtain an acceptable estimation without the incorporation of any image. Nevertheless, one is not able to detect inner parcel changes until the assimilation of new acquisitions. This is the case of the last two estimations without images, i.e., based on prediction (last two empty circles). In this case, the parcel behaves in a different way to the expected by the model and thus the prediction error is larger in those states. This error is reduced significantly when a new image is available, the last one of the series.

The same procedure was applied to all the available set of parcels and images, providing the results shown in Fig. 6, where the estimated results are compared with the ground truth data. In the same way, the empty circles represent unavailable observations (when estimations are based only on the prediction model) and the filled circles are estimations considering both prediction and observation. A coefficient of determination

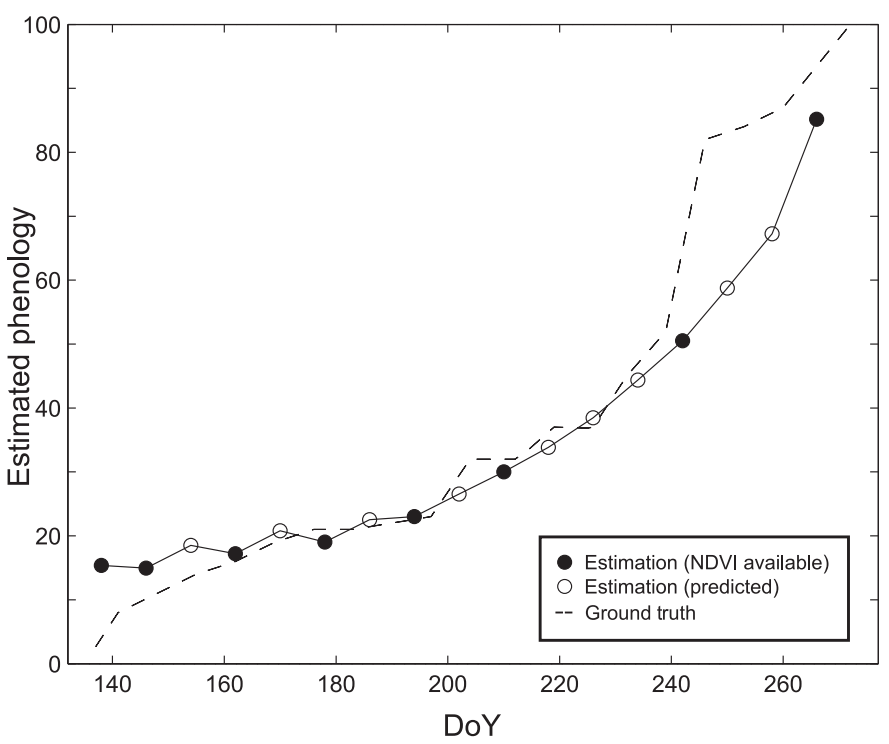

Fig. 5. Phenological state estimates and ground truth for one parcel at 2009 campaign. Estimates are represented by circles: empty circles for estimations based on the prediction and filled ones for estimations combining prediction and NDVI observation. The continuous line represents the linear interpolation between estimates and the dashed line the reference or ground truth data for the parcel.

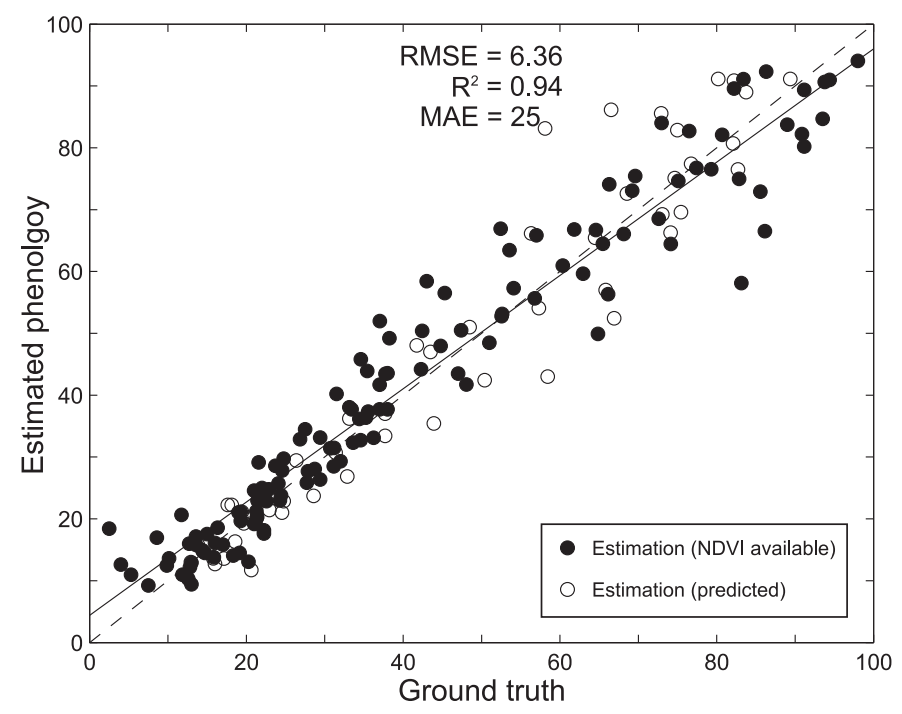

Fig. 6. Phenological state estimates and ground truth for all parcels and available images. The total number of estimates is $n=128,50$ of which are based on prediction only (empty circles) and 78 on prediction and NDVI observation (filled circles). The dashed line represents the identity, and the continuous line is a linear regression to the estimation.

$R^{2}=0.94$ is obtained, with a rmse of 6.36 states and a maximum absolute error (MAE) of 25 states. It is observed that approximately after state 40 dispersion increases. This can be due in part to the fact that the NDVI keeps a constant value around 0.8 within the phenological range from states 40 to 80 (i.e., it is not sensitive to phenological changes in that range) so it cannot improve the accuracy of the prediction.

2) NDVI-SAR Data Fusion: In order to show the enhancement achieved by the fusion approach, the same parcel used in Section IV-A1 is evaluated under the data fusion consideration. 


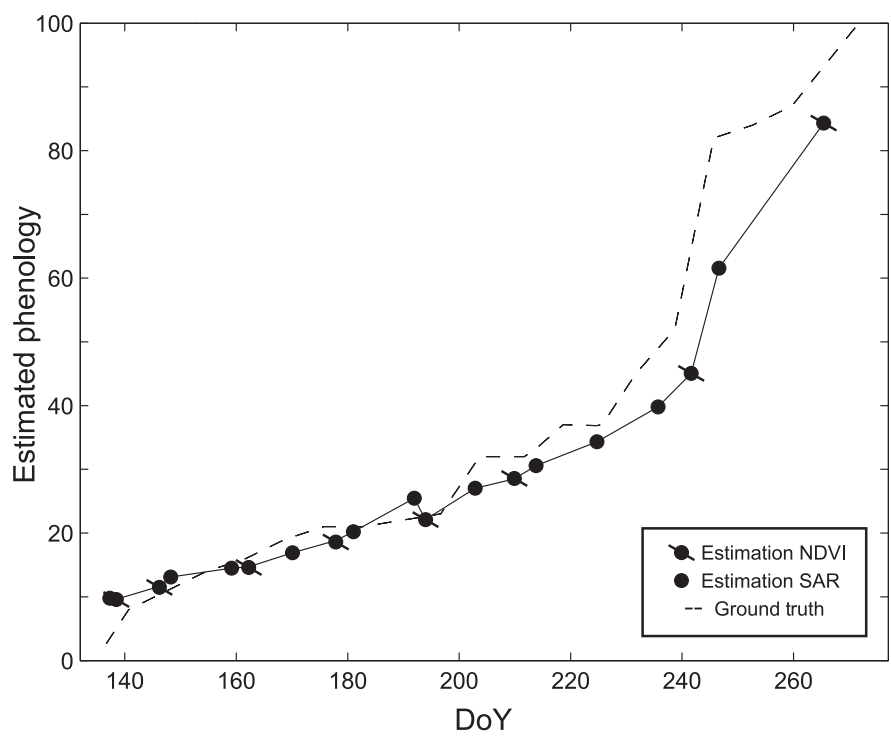

Fig. 7. Phenological state estimation using fusion context (NDVI and SAR) and ground truth for one parcel at 2009 campaign. Estimates are represented by circles, all of them based on prediction and observation. The continuous line represents the linear interpolation between estimates and the dashed line corresponds to the reference or ground truth data for this parcel.

The results are presented in Fig. 7. The estimation is provided each time a SAR or NDVI image, or both are available. The total number of images in this case is 19 ( 8 from the NDVI series and 11 from SAR data set). The obtained rmse is reduced to 7.1 states.

The improvement provided by the incorporation of the SAR acquisitions is noticeable. On the one hand, there is a remarkable increase in the number of observations, from the original set of $8-19$, which doubles the input information. This has a direct benefit over the estimates, as the accumulated error is compensated more often and the gap between estimations is reduced. On the other hand, the convergence time is also reduced. Using exclusively NDVI acquisitions it takes around 20 days in achieve the convergence, while the NDVI-SAR fusion obtains convergent results in just half of the time.

The same estimation procedure is applied to all available parcels using the fusion method. The results are presented in Fig. 8. NDVI and SAR observations are combined to derive an estimation for each parcel, so in this case we show only estimations based on simultaneous prediction and observation (solid circles).

The incorporation of SAR observations improves the results using only optical data, reaching a coefficient of determination $R^{2}=0.96$ with a rmse of 4.40 states and a MAE of 19 states. The most relevant fact is that the dispersion between states 40 and 80 is drastically reduced. Actually, the improvement is even more evident when we focus the analysis in different ranges. In Table II, the specific rmse obtained is disaggregated for different $\mathrm{BBCH}$ ranges. Each row shows the rmse provided by for the corresponding range and using optical data (second column), fusion (third column), and the improvement $\Delta$ (fourth column). In the first stage (0-40), the improvement provided by the SAR observations is only around 1 state. However, in the second stage (40-80), the improvement achieved increases to 3.5 states. The contribution of SAR data is justified in

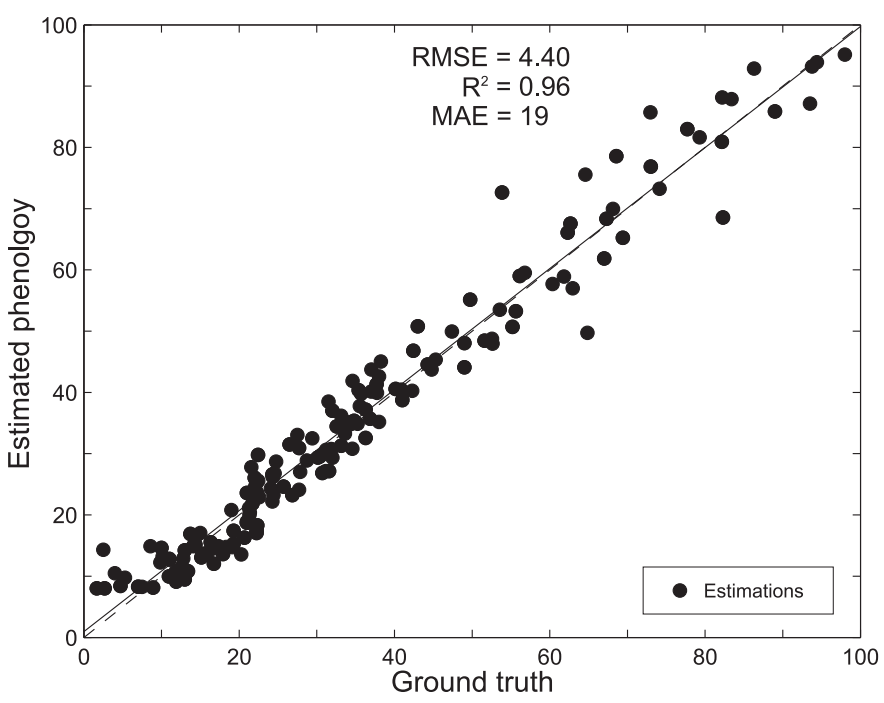

Fig. 8. Phenological estimation using fusion context (NDVI and SAR) for all available parcels and available images. The total number of estimations is $n=$ 163,78 of which are based on NDVI and 85 on SAR observations. The dashed line represents the identity, and the continuous line is a linear regression to the estimation.

TABLE II

RMSE OBTAINED IN SEPARATE BBCH RANGES

\begin{tabular}{cccc}
\hline Range of BBCH & NDVI & NDVI-SAR & $\Delta$ \\
\hline $0-100$ & 6.4 & 4.4 & 2.0 \\
$0-40$ & 4.7 & 3.5 & 1.2 \\
$40-80$ & 9.8 & 6.3 & 3.5 \\
$80-100$ & - & - & - \\
\hline
\end{tabular}

this range, since it provides observations with evolution as a function of phenology, whereas NDVI is mostly constant (not sensitive). In the last stage (80-100), the number of available samples is not enough to provide a feasible value. Except for this range, the statistical significance of the outputs, along with the additional results provided in the study, is granted by $p$-values smaller than 0.0001 . This is a direct result of the large number of available cases.

The inclusion of SAR images has an impact on some important aspects. The first one relies directly on the increase of the number of observations. Therefore, a greater number of events can be observed. The second one is due to the decrease on the time between observations. The effect of the integration of the prediction model derives in a rise of the accumulated error. This error is bounded each time the observation is combined with the predicted value, hence a more often update derives in a decrease in the error of the estimates. And the third aspect relies on the complementarity of both sensors. The SAR acquisitions contribute with higher sensitivity between stages 40 and 80 than the optical sensor, improving the accuracy in this range.

The use of a high number of SAR images could derive significantly in an increase in the cost of the operative final service. In this regard, we could think of alternatives for optimize the number of required images. From our analysis, the inclusion of a smaller number of SAR images located between stages 40 
TABLE III

RESUlTS FOR THE EVALUATION OF RANDOM REMOVAL OF NDVI ACQUISITIONS

\begin{tabular}{c|ccc|ccc}
\hline & \multicolumn{3}{|c}{ NDVI } & \multicolumn{3}{c}{ NDVI-SAR } \\
\hline \# Removed NDVI images & RMSE & $R^{2}$ & MAE & RMSE & $R^{2}$ & MAE \\
\hline 1 & 6.8 & 0.93 & 24 & 4.4 & 0.96 & 19 \\
2 & 7.06 & 0.93 & 24 & 4.35 & 0.96 & 19 \\
3 & 7.62 & 0.92 & 24 & 4.6 & 0.96 & 19 \\
4 & 11.39 & 0.84 & 39 & 4.9 & 0.96 & 19 \\
5 & 12.88 & 0.81 & 43 & 5.0 & 0.95 & 19 \\
6 & 18.15 & 0.74 & 49 & 5.0 & 0.95 & 20 \\
\hline
\end{tabular}

TABLE IV

RESULTS FOR THE EVALUATION OF RANDOM REMOVAL OF SAR ACQUISITIONS

\begin{tabular}{c|ccc|ccc}
\hline & \multicolumn{3}{|c}{ SAR } & \multicolumn{3}{c}{ NDVI-SAR } \\
\hline \# Removed SAR images & RMSE & $R^{2}$ & MAE & RMSE & $R^{2}$ & MAE \\
\hline 1 & 5.2 & 0.94 & 19 & 4.6 & 0.95 & 20 \\
2 & 5.3 & 0.94 & 19 & 4.7 & 0.95 & 20 \\
3 & 5.8 & 0.92 & 24 & 5.0 & 0.94 & 21 \\
4 & 6.4 & 0.90 & 24 & 5.1 & 0.95 & 21 \\
5 & 7.8 & 0.86 & 27 & 5.3 & 0.94 & 22 \\
6 & 10.5 & 0.80 & 31 & 5.7 & 0.93 & 23 \\
\hline
\end{tabular}

and 80 improves the estimation process. The results shown in Fig. 8 are tested again using just two SAR images per parcel, specifically, one acquired around stage 40 and the other around stage 80 . In that case, the attained rmse is 4.9 , thus between the results obtained using the whole set of SAR images and using only NDVI acquisitions. Therefore, even if that is not the optimum result, we have proved that only a small number of SAR images, placed in the proper moment, provide better results than only NDVI images.

The geographic area under test is a particular region with low cloud probability and, therefore, it represents an excellent area to work with optical sensors. However, this may not be the case over other territories with higher probability of clouds, deriving in temporal stacks with erratic gaps or in the extreme case a total lack of multitemporal optical data. So as to evaluate how such scenario affects the proposed approach, the following analysis carried out by removing images from the stack has been performed. First, the impact on the optical domain is studied by excluding randomly NDVI acquisitions. The simulation is repeated 30 times, changing randomly the removed images each time, to be statistically representative. The estimation is carried out with an increasing number of omitted images (from 1 to 6), for both the NDVI series and the NDVI-SAR set. Table III summarizes the results for all cases and data domains. Observing the output of the analysis, it is clear that there is a significant degradation (up to three times) in the rmse when removing data from the NDVI data set when it is the only source of information. In contrast, dropping these same images from the NDVI + SAR data set has a relatively low impact on the rmse (an increase around 0.7 with respect to the whole data set). For instance, when we remove five NDVI images, the results obtained with only the remaining NDVI data provide rmse = 12.9 states, $R^{2}=0.81$, and MAE $=43$ states. In contrast, the fusion with SAR data improves the results in this case to rmse $=5$ states, $R^{2}=0.95$, and MAE $=19$ states.

For completeness, an analog analysis has been engaged from the SAR point of view. The summary in this case is shown in Table IV. Similar insights are provided from the analysis, although some differences can be found. The impact over the SAR series in this case is smaller than over the NDVI images, since dropping a total of six SAR acquisitions only doubles the rmse from the whole data set. Nonetheless, the fused domain is affected more in this case (an rmse increase of 1.1 for six images). This indicates a higher relevance of the SAR data over the fused data results.

\section{B. Results Considering Temperature and Remote Sensing Data}

1) NDVI Time Series: In this section, only the estimation results employing the whole set of parcels is presented. Fig. 9 
TABLE V

Results For the Different Complementary Data EMPloyed in the Estimation Process

\begin{tabular}{l|ccc|ccc|ccc}
\hline \multirow{2}{*}{ Model } & \multicolumn{3}{|c}{ NDVI } & \multicolumn{3}{c}{ NDVI-SAR } & \multicolumn{3}{c}{ SAR } \\
\hline \multirow{3}{*}{ Temporal } & RMSE & $R^{2}$ & MAE & RMSE & $R^{2}$ & MAE & RMSE & $R^{2}$ & MAE \\
\cline { 2 - 10 } Temperature & 6.36 & 0.94 & 25 & 4.40 & 0.96 & 19 & 5.10 & 0.95 & 21 \\
& 5.83 & 0.95 & 19 & 3.90 & 0.97 & 16 & 4.50 & 0.97 & 17 \\
\hline
\end{tabular}

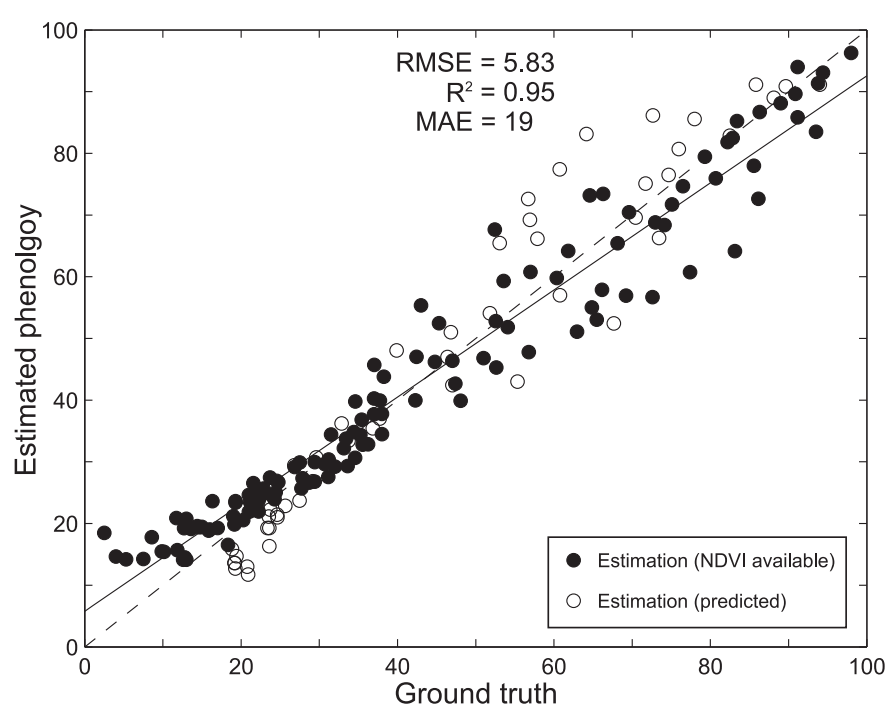

Fig. 9. Phenological state estimates and ground truth for all parcels and available images using the NDVI time-series and $\mathrm{CGD}_{d}$ information. The total number of estimations is $n=128,50$ of which are based on prediction (empty circles), and 78 on temperature-based prediction and NDVI observation (filled circles). The dashed line represents the identity, and the continuous line is a linear regression to the estimation.

shows the estimation outputs using the NDVI time series as observation data and taking the temperature into account in the prediction model. When the observation is unavailable the estimation is obtained only by prediction. The temperature incorporates information in a daily basis, which is used to lead the model. This extra information improves the accuracy in the estimation process. However, the results produced are only slightly better than those shown in Fig. 6 because the years of study present similar temperature behavior. The coefficient of determination is $R^{2}=0.95$, with an rmse of 5.83 states and a MAE of 19 states.

2) NDVI-SAR Data Fusion: To complete the analysis, all the available information (NDVI, SAR, and $\mathrm{CGD}_{d}$ ) are used in the estimation. NDVI and SAR images are incorporated in the observation step and temperature data in the prediction step. The results are illustrated in Fig. 10. The prediction step is driven by the temperature providing the prior pdf. Then, when an observation is available (SAR, NDVI, or both), the prior is updated by the likelihood function. In this case, the incorporation of the three different sources of information yields a coefficient of determination $R^{2}=0.97$ with an rmse of 3.90 states and a MAE of 16 states. The high accuracy is due to the fact that both steps (prediction and observation) are improved.

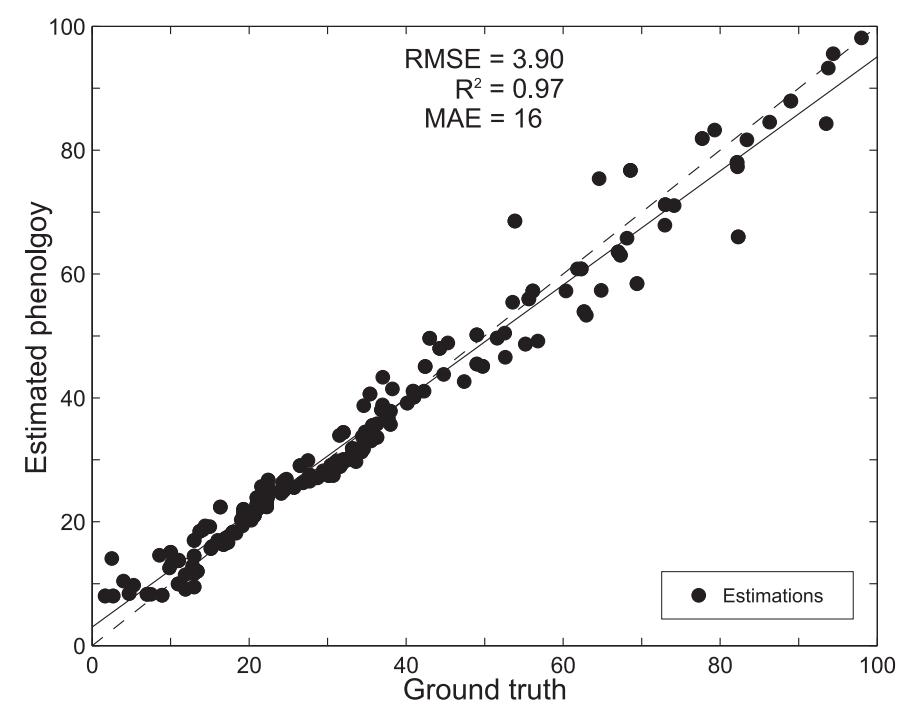

Fig. 10. Phenological estimation using fusion context (NDVI and SAR) and $\mathrm{CGD}_{d}$ information for all available parcels and available images. The total number of estimations is $n=163,78$ of which are based on NDVI and 85 on SAR observations. The dashed line represents the identity, and the continuous line is a linear regression to the estimation.

As a summary of all results, the rmse obtained for all cased is presented in Table V. The first row shows the results using the temporal model, and in the second one the temperature is taken into account in the prediction step. The first group of columns present the accuracy of the results of the estimation considering observations just made by the NDVI time series and the second considering also the SAR data information in a fusion context. Finally, the third column shows the results considering only SAR observations. A deep evaluation of this case is not considered in this work but is summarized here to complete the global results. More information about the estimation of phenological state using SAR observations can be found in a previous work [20]. It should be noticed that the rmse with SAR observations improves the obtained with NDVI images. This is mainly due to a regular temporal sampling in SAR (for this case 11 days) and the higher sensitivity in the specific range from 40 to 60 . Predictions are updated every 11 days whereas with NDVI images the time between acquisitions cannot be guaranteed. The temporal sampling of NDVI data (for this case) is 8 days by default, but the presence of cloud worsens it to 32 days in some periods. In any case, the fusion methodology produces the best results and the temperature information also improves the estimation, achieving an RSME of 3.90. In terms of the MAE, the lowest value is also obtained with this complete configuration. 


\section{CONCLUSION}

In this study, we have developed and evaluated a formulation for the incorporation of additional sources of information to a dynamical methodology for crop phenological estimation. In particular, a methodology based on the PF algorithm, previously defined to exploit time series of NDVI data, has been complemented with SAR acquisitions and temperature data. The estimation framework enables providing phenology estimates in an online manner, i.e., as a real-time application, with evident benefits for the final users.

Including additional sources to the NDVI, time series has shown that it is possible to increase in the sensitivity to the phenological evolution. On the one hand, with the incorporation of SAR acquisitions, variations from states 40 to 80 can be detected better than using only NDVI data. That supports how this alternative technology represents a valuable complement to the multispectral product. Moreover, the total number of observations is increased, reducing the temporal acquisition period, and subsequently improving the tracking of the crop dynamical development. The improvement provided by data fusion is especially noticeable when the number of NDVI images is low. On the other hand, the use of temperature has shown a slight improvement in the estimation process. In this case, the small temperature variations considered during the generation of the prediction model limit importantly the contribution of this variable. It can be also interpreted as if the effects of the temperature were already incorporated in the temporal model. From our analysis, we can conclude that the reliance on different sources of information allows us to derive more solid outcomes than strategies based on a single data source.

Ongoing works are addressed to increase the value of the estimation results and to provide a reliable operational product. From the methodological point of view, some considerations can be addressed to define a multidimensional observation space (considering all bands from multispectral sensors and/or all polarimetric channels from a SAR), which could increase the sensitivity to all the phenological range. In order to fully validate the potential of the contribution of the temperature, an extended analysis employing a representative data set has also to be considered.

From the application point of view future evaluations should include alternative study areas and derive the capability to detect cultivation problems, such us plagues or nutrient deficits. For this purpose, the impact of the resolution on the algorithm performance should be studied. It would be interesting to analyze in a future work how to extract the optimal resolution (or the integration) of different resolution remote sensing products. Finally, the evaluation of the methodology over other type of crops, such as cereals, should be carried out to prove the whole potential of the proposed framework.

\section{ACKNOWLEDGMENT}

The authors would like to thank the support of the Federación de Arroceros de Sevilla for providing the ground measurement data and for their helpful comments. All NDVI images employed in this work were derived from images acquired by satellites Landsat 5 and Landsat 7 . Images were freely downloaded from the Servidor de imágenes Landsat y productos derivados de Doñana, a web server (http://mercurio.ebd.csic.es/imgs/) maintained and operated by the Laboratorio de Sistemas de Información Geográfica y Teledetección de la Estación Biológica de Doñana, LASTEBD, CSIC, Spain. All SAR images have been provided by DLR in the framework of projects LAN0021 and LAN0234 of the prelaunch AO of TerraSAR-X. Temperature information obtained from the Sistema de Información Agroclimática para el Regadio (SIAR), Spanish Ministry of Agriculture, Food and Environment.

\section{REFERENCES}

[1] A. S. Islam and S. K. Bala, "Assessment of potato phenological characteristics using MODIS-derived NDVI and LAI information," GISci. Remote Sens., vol. 45, no. 4, pp. 454-470, 2008.

[2] T. Sakamoto, M. Yokozawa, H. Toritani, M. Shibayama, N. Ishitsuka, and H. Ohno, "A crop phenology detection method using time-series MODIS data," Remote Sens. Environ., vol. 96, no. 3, pp. 366-374, 2005.

[3] B. D. Wardlow, J. H Kastens, and S. L. Egbert, "Using USDA crop progress data for the evaluation of greenup onset date calculated from MODIS 250-meter data," Photogramm. Eng. Remote Sens., vol. 72, no. 11, pp. 1225-1234, 2006.

[4] H. Fan, X. Fu, Z. Zhang, and Q. Wu, "Phenology-based vegetation index differencing for mapping of rubber plantations using Landsat OLI data," Remote Sens., vol. 7, no. 5, pp. 6041-6058, 2015.

[5] P. J. Sellers, J. A. Berry, G. J. Collatz, C. B. Field, and F. G. Hall, "Canopy reflectance, photosynthesis, and transpiration. III. A reanalysis using improved leaf models and a new canopy integration scheme," Remote Sens. Environ., vol. 42, no. 3, pp. 187-216, 1992.

[6] S. W. Running, "Estimating terrestrial primary productivity by combining remote sensing and ecosystem simulation," in Remote Sensing of Biosphere Functioning. New York, NY, USA: Springer, 1990, pp. 65-86.

[7] R. B. Myneni and F. G. Hall, "The interpretation of spectral vegetation indexes," IEEE Trans. Geosci. Remote Sens., vol. 33, no. 2, pp. 481-486, Mar. 1995.

[8] J. Zhang, "Multi-source remote sensing data fusion: Status and trends," Int. J. Image Data Fusion, vol. 1, no. 1, pp. 5-24, 2010.

[9] F. Bovolo and L. Bruzzone, "The time variable in data fusion: A change detection perspective," IEEE Geosci. Remote Sens. Mag., vol. 3, no. 3, pp. 8-26, Sep. 2015.

[10] F. Orsomando, P. Lombardo, M. Zavagli, and M. Costantini, "SAR and optical data fusion for change detection," in Proc. IEEE Urban Remote Sens. Joint Event, 2007, pp. 1-9.

[11] A. Salentinig and P. Gamba, "Combining SAR-based and multispectralbased extractions to map urban areas at multiple spatial resolutions," IEEE Geosci. Remote Sens. Mag., vol. 3, no. 3, pp. 100-112, Sep. 2015.

[12] B. Waske, J. A. Benediktsson, and J. R. Sveinsson, "Fusion of multisource data sets from agricultural areas for improved land cover classification," in Proc. IEEE Int. Geosci. Remote Sens. Symp. (IGARSS'09), Jul. 2009, vol. 4, pp. IV-952-IV-955.

[13] F. Tupin and M. Roux, "Markov random field on region adjacency graph for the fusion of SAR and optical data in radargrammetric applications," IEEE Trans. Geosci. Remote Sens., vol. 43, no. 8, pp. 1920-1928, Aug. 2005.

[14] F. Gao et al., "Fusing Landsat and MODIS data for vegetation monitoring," IEEE Geosci. Remote Sens. Mag., vol. 3, no. 3, pp. 47-60, Sep. 2015.

[15] N. D. A. Mascarenhas, G. J. F. Banon, and A. L. B. Candeias, "Multispectral image data fusion under a Bayesian approach," Int. J. Remote Sens., vol. 17, no. 8, pp. 1457-1471, 1996.

[16] A. Stein, "Use of single-and multi-source image fusion for statistical decision-making," Int. J. Appl. Earth Observ. Geoinf., vol. 6, no. 3, pp. 229-239, 2005.

[17] J. A. Benediktsson, P. H. Swain, and O. K. Ersoy, "Neural network approaches versus statistical methods in classification of multisource remote sensing data," in Proc. EEE Trans. Geosci. Remote Sens., 1989, pp. 489-492. 
[18] G. G. Rigatos, "Extended Kalman and particle filtering for sensor fusion in motion control of mobile robots," Math. Comput. Simul., vol. 81, no. 3, pp. 590-607, 2010.

[19] F. Vicente-Guijalba, T. Martinez-Marin, and J. M. Lopez-Sanchez, "Crop phenology estimation using a multitemporal model and a Kalman filtering strategy," IEEE Geosci. Remote Sens. Lett., vol. 11, no. 6, pp. 1081-1085, Jun. 2014

[20] C. De Bernardis, F. Vicente-Guijalba, T. Martinez-Marin, and J. M. Lopez-Sanchez, "Estimation of key dates and stages in rice crops using dual-polarization SAR time series and a particle filtering approach," IEEE J. Sel. Topics Appl. Earth Observ. Remote Sens., vol. 8, no. 3, pp. 1008-1018, Mar. 2015.

[21] C. De Bernardis, F. Vicente-Guijalba, T. Martinez-Marin, and J. M. Lopez-Sanchez, "Particle filter approach for crop phenological stage estimation using time series of NDVI images," in Proc. IEEE Int. Geosci. Remote Sens. Symp. (IGARSS), 2015, pp. 3385-3388.

[22] U. Meier, Ed., Growth Stages of Mono and Dicotyledonous Plants. $B B C H$ Monograph, 2nd ed. Berlin, Germany: Federal Biological Research Centre for Agriculture and Forestry, 2001 [Online]. Available: http://www.jki.bund.de/fileadmin/dam_uploads/_veroeff/bbch/BBCHSkala_englisch.pdf

[23] A. F Hunter and M. J. Lechowicz, "Predicting the timing of budburst in temperate trees," J. Appl. Ecol., vol. 29, pp. 597-604, 1992

[24] C. Galán, H. García-Mozo, P. Cariñanos, P. Alcázar, and E. DomínguezVilches, "The role of temperature in the onset of the Olea europaea L. pollen season in southwestern Spain," Int. J. Biometeorol., vol. 45, no. 1, pp. 8-12, 2001.

[25] M. Boschetti, D. Stroppiana, P. A. Brivio, and S. Bocchi, "Multi-year monitoring of rice crop phenology through time series analysis of MODIS images," Int. J. Remote Sens., vol. 30, no. 18, pp. 4643-4662, 2009.

[26] R. Confalonieri and S. Bocchi, "Evaluation of CropSyst for simulating the yield of flooded rice in northern Italy," Eur. J. Agron., vol. 23, no. 4, pp. 315-326, 2005.

[27] R. Confalonieri, M. Acutis, G. Bellocchi, and M. Donatelli, "Multi-metric evaluation of the models WARM, CropSyst, and WOFOST for rice," Ecol. Modell., vol. 220, pp. 1395-1410, 2009.

[28] A. Doucet, S. Godsill, and C. Andrieu, "On sequential Monte Carlo sampling methods for Bayesian filtering," Stat. Comput., vol. 10, no. 3, pp. 197-208, 2000.

[29] M. H. Kalos and P. A. Whitlock, Monte Carlo Methods. Hoboken, NJ, USA: Wiley, 2008

[30] M. S. Arulampalam, S. Maskell, N. Gordon, and T. Clapp, "A tutorial on particle filters for online nonlinear/non-Gaussian Bayesian tracking," IEEE Trans. Signal Process., vol. 50, no. 2, pp. 174-188, Feb. 2002.

[31] J. W. Enz, A. Bauer, C.V. Eberlein, and C. Fanning, "Use of growingdegree days to determine spring wheat growth stages," North Dakota State Univ. Extension Bull., vol. 37, pp. 1-11, 1984.

[32] S. R. Cloude and E. Pottier, "A review of target decomposition theorems in radar polarimetry," IEEE Trans. Geosci. Remote Sens., vol. 34, no. 2, pp. 498-518, Mar. 1996.

[33] J. M. Lopez-Sanchez, F. Vicente-Guijalba, J. D. Ballester-Berman, and S. R. Cloude, "Polarimetric response of rice fields at C-band: Analysis and phenology retrieval," IEEE Trans. Geosci. Remote Sens., vol. 52, no. 5, pp. 2977-2993, May 2014.

[34] J. M. Lopez-Sanchez, S. R. Cloude, and J. D. Ballester-Berman, "Rice phenology monitoring by means of SAR polarimetry at X-band," IEEE Trans. Geosci. Remote Sens., vol. 50, no. 7, pp. 2695-2709, Jul. 2012.

[35] A. Bouvet, T. Le Toan, and N. Lam-Dao, "Monitoring of the rice cropping system in the Mekong delta using ENVISAT/ASAR dual polarization data," IEEE Trans. Geosci. Remote Sens., vol. 47, no. 2, pp. 517-526, Feb. 2009.

[36] J. Peñuelas, I. Filella, and P. Comas, "Changed plant and animal life cycles from 1952 to 2000 in the Mediterranean region," Global Change Biol., vol. 8, no. 6, pp. 531-544, 2002.

[37] X. Zhang et al., "Monitoring vegetation phenology using MODIS," Remote Sens. Environ., vol. 84, no. 3, pp. 471-475, 2003.

[38] J. Bustamante, F. Pacios, R. Díaz-Delgado, and D. Aragonés, "Predictive models of turbidity and water depth in the Doñana marshes using Landsat TM and ETM+ images," J. Environ. Manage., vol. 90, pp. 2219-2225, 2009.

[39] R. Díaz-Delgado, D. Aragonés, I. Ameztoy, and J. Bustamante, "Monitoring marsh dynamics through remote sensing," in Conservation Monitoring in Freshwater Habitats: A Practical Guide and Case Studies. New York, NY, USA: Springer, 2010, pp. 325-337.

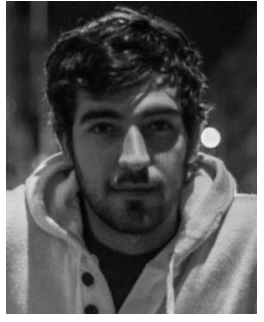

Caleb De Bernardis was born in Mar del Plata, Buenos Aires, Argentina, in 1989. He received the Ingeniero Tecnico (B.S.) degree (summa cum laude) in sound and image engineering from the University of Alicante, Alicante, Spain, in 2010, and the Ingeniero (M.S.) degree in telecommunication engineering from the Technical University of Valencia (UPV), Valencia, Spain, in 2014. He is currently pursuing the $\mathrm{Ph} . \mathrm{D}$. degree in computer science at Signals, Systems, and Telecommunications Group, University of Alicante. He collaborated with the Nanophotonics Technology Centre (NTC), Valencia, Spain, in 2013.

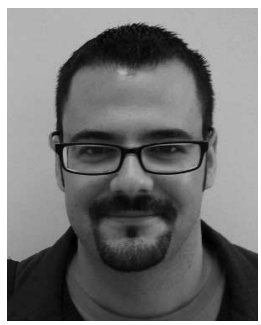

Fernando Vicente-Guijalba was born in Elche, Alicante, Spain, in 1981. He received the Tech. Eng. (B.S.) and M.S. degrees in telecommunications engineering from the University of Alicante, Alicante, Spain, in 2006 and 2014, respectively.

He has been a Predoctoral Fellow with the Signals, Systems, and Telecommunications Groups, University of Alicante, since 2012. His research interests include dynamical systems analysis with applications in the polarimetric and interferometric SAR methods.

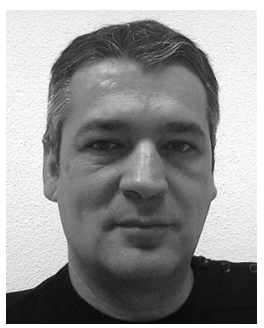

Tomas Martinez-Marin received the Tech. Eng. (B.S.) degree in in telecommunication engineering from the University of Alcalá (UAH), Alcalá de Henares, Spain, in 1990, and the M.S. and the Ph.D. degrees in telecommunications engineering from the Technical University of Madrid (UPM), Madrid, Spain, in 1995 and 1999, respectively.

He joined the University of Alcalá as an Assistant Professor in 1990. In 1997, he joined European University of Madrid (UEM), Madrid, Spain, as an Assistant Professor. Since 2000, he has been with the Department of Physics, System Engineering and Signal Theory, University of Alicante (UA), Alicante, Spain, where he is currently an Associate Professor. His research interests include reinforcement learning, optimal control, intelligent vehicles, and SAR filtering algorithms.

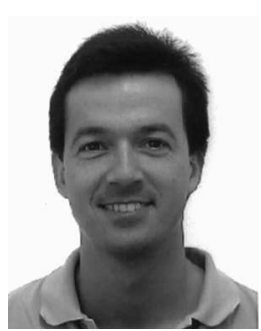

Juan M. Lopez-Sanchez (S'94-M'00-SM'05) was born in Alicante, Spain, in 1972. He received the Ingeniero (M.S.) and Doctor Ingeniero (Ph.D.) degrees in telecommunication engineering from the Technical University of Valencia (UPV), Valencia, Spain, in 1996 and 2000, respectively.

From 1998 to 1999, he was a Predoctoral Grantholder of the Space Applications Institute, Joint Research Centre of the European Commission, Ispra, Italy. Since 2000, he has been leading the Signals, Systems, and Telecommunication Group, University of Alicante, Alicante, Spain, where he has been a Full Professor since November 2011. He has coauthored more than 55 papers published in refereed journals and more than 100 papers and presentations published in international conferences and symposia. His research interests include microwave remote sensing for inversion of biophysical parameters, polarimetric and interferometric techniques, SAR imaging algorithms, and applications of radar remote sensing in agriculture and geophysics.

Dr. Lopez-Sanchez was the Chair of Spanish Chapter of the IEEE Geoscience and Remote Sensing Society, from 2006 to 2012. He was the recipient of the Indra Award for the Best Ph.D. Thesis About Radar in Spain, in 2001. 